\title{
Vision metrology and Structure from Motion for archaeological heritage 3D reconstruction: a Case Study of various Roman mosaics
}

\author{
Mauro Lo Brutto, Gino Dardanelli \\ Dept. of Civil, Environmental, Aerospace, Materials Engineering (DICAM), University of Palermo, viale delle Scienze, 90128 Palermo, Italy
}

\begin{abstract}
Vision metrology and computer vision can be successfully used for archaeological heritage 3D reconstruction in very high uncertainty 3D measurement projects. Of those archaeological objects requiring very accurate measurements ( $<1 \mathrm{~mm})$, ancient mosaics comprise some of the most important. The aim of this paper is to assess the photogrammetric/computer vision approach in a vision metrology context as part of a 3D mosaics survey. In order to evaluate the optimal photogrammetric/computer vision workflow in this work, three different surveys were performed on three mosaics of different sizes and locations. Two of these are stored at the Antonino Salinas Regional Archaeological Museum in Palermo (Italy) and the other is located at the Baglio Anselmi Regional Archaeological Museum in Marsala (Italy). The mosaics survey was undertaken in order to obtain a very detailed 3D model and a full-scale orthoimage (scale 1:1), which would be useful for documentation and restoration processes. The research involved an evaluation of the potential and the related issues of the photogrammetric/computer vision approach for 3D mosaic documentation, particularly regarding the issue of camera calibration.
\end{abstract}

\section{Section: RESEARCH PAPER}

Keywords: mosaic; photogrammetry; computer vision; camera calibration; 3D model; accuracy

Citation: Mauro Lo Brutto, Gino Dardanelli, Vision metrology and Structure from Motion for archaeological heritage 3D reconstruction: a Case Study of various Roman mosaics, Acta IMEKO, vol. 6, no. 3, article 6, September 2017, identifier: IMEKO-ACTA-06 (2017)-03-06

Section Editor: Sabrina Grassini, Politecnico di Torino, Italy

Received March 3, 2017; In final form June 15, 2017; Published September 2017

Copyright: (C) 2017 IMEKO. This is an open-access article distributed under the terms of the Creative Commons Attribution 3.0 License, which permits unrestricted use, distribution, and reproduction in any medium, provided the original author and source are credited

Corresponding author: Mauro Lo Brutto, e-mail: mauro.lobrutto@unipa.it

\section{INTRODUCTION}

A 3D survey of archaeological heritage is a fundamental step in increasing our knowledge of archaeological finds and sites. Much information can be obtained from a correct 3D documentation, including: measurements regarding the shape and size of objects, information relating to the state of preservation, data related to material deterioration, etc.

The techniques available to us as part of a $3 \mathrm{D}$ survey of cultural archaeological heritage permit us to obtain very accurate and detailed metric data for large and small dimension objects [1]. In archaeology a 3D survey of objects requiring a very high level of accuracy $(<1 \mathrm{~mm})$ can be performed typically with active sensors (such as triangulation-based range sensors and pattern projection sensors) or passive sensors (such as image-based techniques) [2]. In recent years, image-based techniques have become increasingly used in 3D surveys, thanks to the integration of a photogrammetric and computer vision approach. This integration has enabled the development of a fully automated pipeline. Some examples are reported in [3] where the advantage of photogrammetric/computer vision approach is demonstrated for stratigraphic archaeological excavations. This work outlines how such approach can be applied to map archaeological excavations in a very fast and uncomplicated way, using imagery shot with a standard compact digital camera (even if the images were not taken for this purpose). Photogrammetric and computer approach in archaeology has become increasingly popular due mainly to the availability of abundant low-cost and open-source software and the ease of processing stages. In [4] some well-known opensource software packages, a web service and a low-cost software for image-based generation of point clouds or $3 \mathrm{D}$ mesh were compared in order to analyse the accuracy and the 
reliability of 3D model of archaeological finds and objects. Further tests have been done using web service in [5] for 3D modelling of architectural heritage. Some researchers have aimed at investigating the potentials of the computer visionbased software package Agisoft PhotoScan, for the 3D documentation of archaeological surfaces and contexts during excavations, for the visualisation of excavation data during the post-excavation process and for the visualisation of the unmovable archaeological heritage for a professional and a wider audience [6]. Furthermore, several recent studies have demonstrated that photogrammetric and computer vision approach has improved its performance to the point of being comparable with that of active range sensors [7], [8].

As is widely known, one of the main objectives of photogrammetry is measurement accuracy and, for this reason, the use of close-range photogrammetry in metrology applications is not new. The term vision metrology is also often used to describe this technology, when applied to tasks involving higher accuracy 3D measurement [9]. For example, vision metrology is typically applied to engineering and manufacturing contexts where it is necessary to obtain a measuring accuracy in the range of a few tens of micrometres to tenths of a millimeter, and where object size is in the range $1 \div 10$ m [10]. Vision metrology applications could be for quality control [11], for reverse engineering [12] or for soil microtopography evaluation [13], [14]. The measurement results must be accurate, repeatable and traceable to national or international standards (such as the German standard VDI/VDE guidelines) [15]. To achieve such a degree of performance, vision metrology systems adopt carefully designed image networks, calibrated cameras, coded targets for point measurements and very accurate scale bars to scale the photogrammetric model and to check for accuracy [16]. Computer vision, however, aims at the automatic image orientation of large unordered and uncalibrated image sequences, using a Structure for Motion (SfM) algorithm [17] and it does not place any emphasis on measurement accuracy.

Photogrammetry and computer vision enable us to produce final products with comparable features; originally, computer vision, and particularly the SfM approach, were not considered suitable for application to vision metrology due to a lack of results in terms of accuracy and the reliability of the process. Recently, advances have been made in several software packages that permit the integration of the SfM and photogrammetric approach into the same pipeline. Examples of these include: commercial software packages like Agisoft Photoscan, Pix4D, ContexCapture, 3DF Zephyr or open source software packages like MicMac. Thus, the photogrammetric/computer vision approach could also be deployed in a metrological context, even if many aspects (like the camera network or the camera calibration) must be taken into account. A number of tests have been reported in a metrological context for both outdoor and environmentally controlled conditions [18] and for geoscience applications [19].

In a 3D survey of archaeological heritage, there are various circumstances in which the metrological aspect is very important: for example, the survey and documentation of ancient mosaics, ancient paintings and frescoes requires submillimetre uncertainty with which to plan a restoration and conservation activities [20], [21]. These objects will have to be measured and monitored to a high level of accuracy because small geometric variations could cause very serious damage.
Of those objects requiring very accurate measurements $(<1$ $\mathrm{mm})$, mosaics are a special case due to their variability in size (mosaics can range from a few decimetres to several metres) and the diverse conditions of conservation (the mosaics can be in an original site or preserved in a museum). Moreover, the presence of the tesserae, the reference unit of these objects, renders a mosaics survey challenging. Although the dimensions of the mosaics may be measured in metres, the single tessera is always very small (generally a few millimetres). The tesserae of ancient mosaics are generally unique elements, which are characterized by a high geometric and material complexity. The traditional survey approach involves single tessera, using a transparent polyester film that rests in contact with the mosaic. All the tesserae are drawn on the film in order to obtain a fullscale (scale 1:1) drawing. Whilst extremely detailed and accurate, this type of documentation has several drawbacks: the lengthy time required for drawing, the difficulty of operating in situ and operator subjectivity. In addition, any drawing produced is unwieldy to use and reproduce, and not easily portable due to its size.

The use of image-based techniques is a great support in surveying mosaics, specifically regarding the documentation of single tesserae and a full-scale (scale 1:1) drawing. Various applications have been performed in recent years as part of a close-range photogrammetry survey of the mosaics of Saint Mark's Basilica in Venice [22]. In this work, the researchers obtained a 3D model and an ortho-image on a scale of 1:1 of the entire mosaic of the Basilica $\left(2,100 \mathrm{~m}^{2}\right)$, using a traditional close-range photogrammetric approach. More recently, additional tests have been undertaken in order to compare different mosaic point clouds, from laser scanners and from SfM. This has enabled the documentation of three ancient Roman mosaics and the evaluation of the best point cloud resolution for a detailed shape analysis of each tessera [23].

The aim of this paper is to define the workflow of a mosaic survey, using the photogrammetric/computer vision approach in a vision metrology context. An accuracy assessment relating to the various camera calibration processes has also been performed. The work involved a 3D survey of three ancient Roman mosaics, which were used as test data, and it permitted us to obtain 3D models and ortho-images of the mosaics to a high level of accuracy. Such products could assist in the restoration and preservation process. The paper is organized as follows: Section 2 provides the main information relating to the three ancient mosaics herein discussed. Section 3 describes data acquisition. Section 4 analyses data processing and accuracy results. Section 5 describes $3 \mathrm{D}$ mosaic reconstruction phases. The concluding section contains comments.

\section{THE ANCIENT MOSAICS}

The research involved the use of three ancient Roman mosaics as test data. Two mosaics are preserved at the Antonino Salinas Regional Archaeological Museum in Palermo (Italy) and the third is located at the Baglio Anselmi Regional Archaeological Museum in Marsala (Italy).

The former two mosaics belong to the Piazza della Vittoria archaeological site, which is located within the city centre of Palermo (Italy). The discovery of this archaeological site in one of the oldest and most central parts of Palermo has revealed two buildings: Building $\mathrm{A}$ and Building $\mathrm{B}$. The construction of the former dates back to the early 3rd century $\mathrm{AD}$, that is, the Roman imperial age; the latter can be dated to the late 2 nd 
century BC, that is, the Hellenistic period. The visible remains today of Building A are composed of parts of walls and decorations, which were unearthed during archaeological excavations. Building $\mathrm{A}$ is characterized as having rich mosaic pavements, which were removed and preserved in part in the Antonino Salinas Regional Archaeological Museum and in part left in situ. The two mosaics discussed in this paper belong to Building A.

The first mosaic is a bichrome opus tessellatum mosaic with geometric decorations (Figure 1). The mosaic is made of square tesserae of black and white marble. The mosaic decoration is a geometric drawing, obtained by the arrangement of black tesserae on a white background. The black tesserae are arranged to form rows of recumbent, spindle-shapes that constitute a grid with squares whose sides' measure approximately 40 centimetres; each square has a crosslet with chevrons in the centre. The mosaic is about $5.50 \mathrm{~m}$ by $4.20 \mathrm{~m}$.

The second mosaic is a geometric polychrome mosaic, composed of different colour tesserae (Figure 2). The basic element of the mosaic decoration is the lozenge shape with different colour tesserae arranged to form this repeating, geometric element; thus a slanting direction is formed which

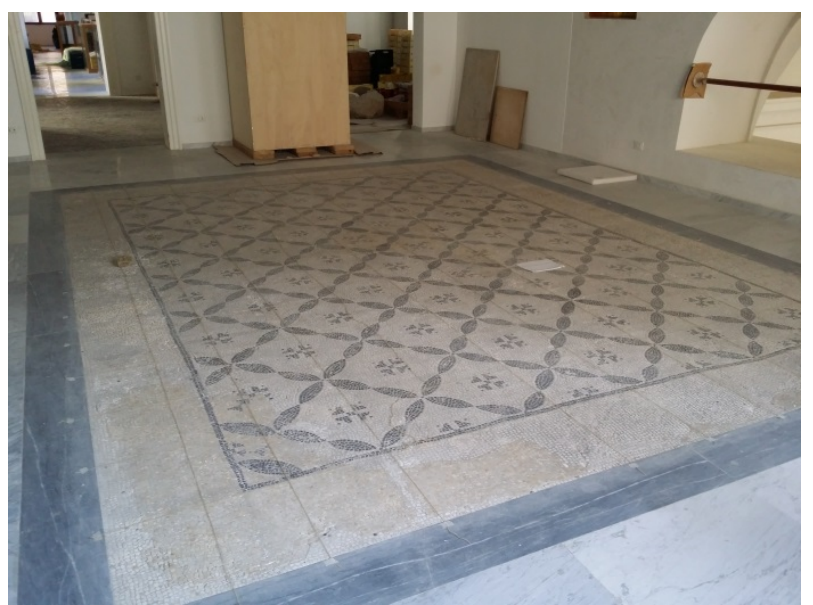

Figure 1. The bichrome opus tessellatum mosaic at the Antonino Salinas Regional Archaeological Museum in Palermo (Italy).

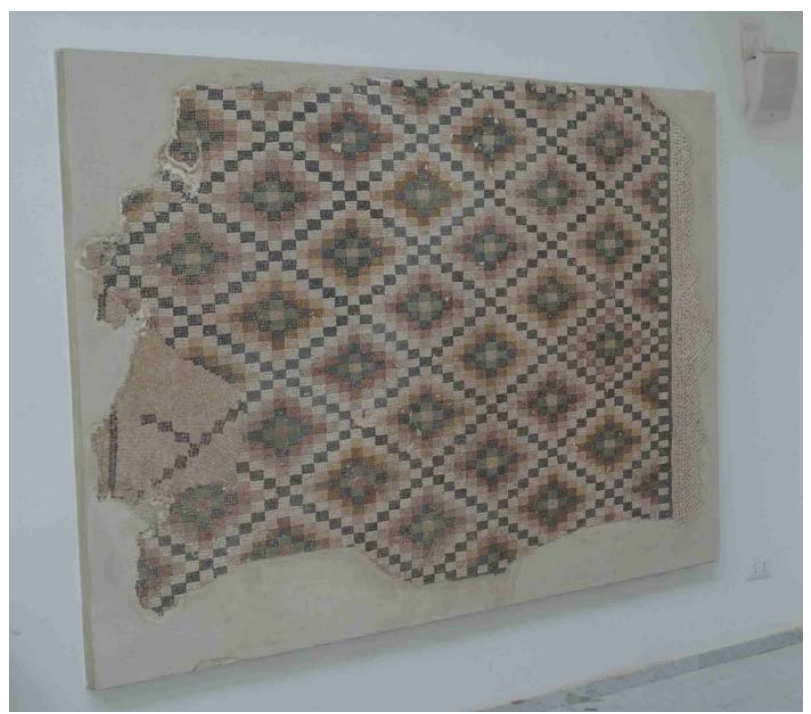

Figure 2. The geometric polychrome mosaic at the Antonino Salinas Regional Archaeological Museum in Palermo (Italy). creates a regular pattern. The materials used for the mosaic are white, black, green, yellow, orange, pink and red limestone. This mosaic is approximately $2.30 \mathrm{~m}$ by $1.75 \mathrm{~m}$.

The third mosaic (Figure 3), preserved at the Baglio Anselmi Regional Archaeological Museum in Marsala (Italy), belongs to the Capo Boeo archaeological site. This archaeological area extends for 28 hectares within the new city of Marsala, much of it overlapping the settlement of the ancient city of Lilybaeum. The Carthaginians founded the latter in the 4th century BC and it later became a Roman colony. The archaeological surveys have uncovered: ancient fortifications, the thermae (baths), the decumanus maximus (the main east-west-oriented road in a Roman city), and various examples of domus (the private homes in a Roman city). The mosaic had originally been placed in compartment n. 36 of a domus in the I insula; it is considered to be the most important due to its considerable architectural complexity and elaborate decoration. The fine details of this mosaic render it a most refined mosaic, characterized by different colours, which paint a geometric polychrome decorative motif. Its dimensions are $4.50 \mathrm{~m}$ by $5.50 \mathrm{~m}$.

\section{DATA ACQUISITION}

The data acquisition has been carried out with the same parameters (same camera, same focal lens, same camera network, same camera-to-object distance, etc.) in order to obtain similar datasets for the three mosaics.

In all three locations, the logistic conditions have not presented particular problems for the photogrammetric survey; to get the best lighting conditions the photogrammetric survey was performed without the use of artificial lights or spotlights but only using natural light.

The images acquisition has been carried out using a Nikon D5200 digital camera equipped with a $28 \mathrm{~mm}$ Nikkor AF-S $\mathrm{f} / 2.8 \mathrm{G}$ fixed focus lens; the camera has a CCD sensor with size of $23.5 \mathrm{~mm} \times 15.7 \mathrm{~mm}$, a pixel size of $3.9 \mu \mathrm{m}$ and an effective resolution of 6000 pixels $\times 4000$ pixels. The camera-to-object distance was chosen equal to $1.5 \mathrm{~m}$; the image scale was $1 / 54$ and the coverage of each image was about $1.2 \mathrm{~m} \times 0.8 \mathrm{~m}$. Because the camera focal length was $28 \mathrm{~mm}$, each pixel was about to $0.2 \mathrm{~mm}$ in the object space.

During the images acquisition the lens was set to manual

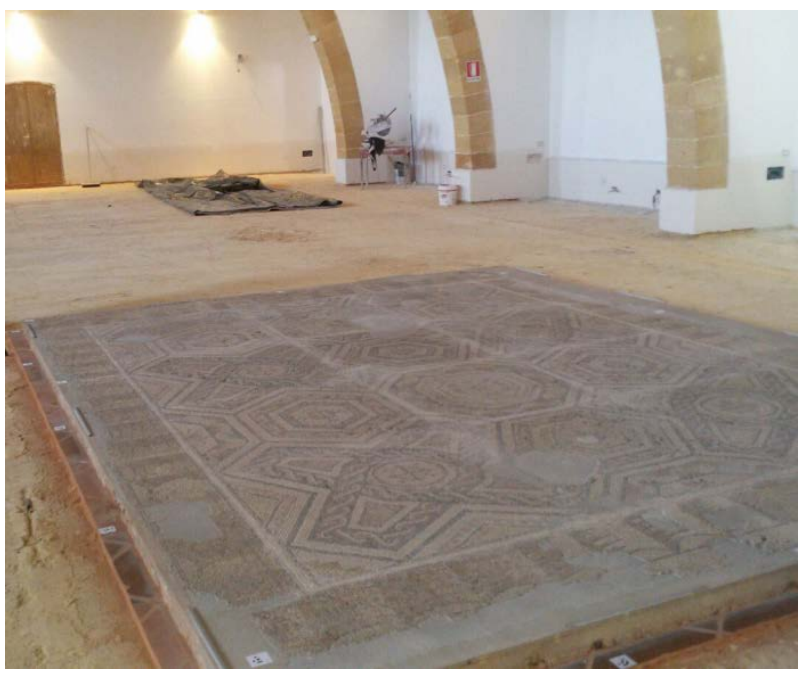

Figure 3. The geometric polychrome mosaic at the Baglio Anselmi Regional Archaeological Museum in Marsala (Italy). 
focus after adjusting it to the average camera-to-object distance of the project and then adequately fixed throughout the shooting process.

A nadiral stereoscopic coverage has been planned for the three mosaics with strips parallel to the longer side of the mosaic. The photogrammetric strips have been selected providing a forward overlap and a lateral overlap of $70 \%$. Some additional convergent strips have been also planned along the edge of the mosaic to increase the redundancy of the measures at the edges of the photogrammetric block and to limit bowl-effect in the 3D model [24]. In this way three photogrammetric datasets have been obtained, called "Mosaic1 " for the first mosaic, "Mosaic-2" for the second and "Mosaic3" for the third. Figure 4 shows the camera network for the three datasets. The images have been taken with the camera mounted on a tripod (Figure 5); this condition has allowed to acquire nadiral images maintained the sensor position parallel to mosaic's plane.

During data acquisition, some extra convergent images were also taken; overall Mosaic- 1 and Mosaic- 3 have almost the same number of images, Mosaic- 2 has about a quarter of images than the others (Table 1).

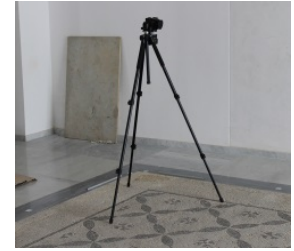

(a)

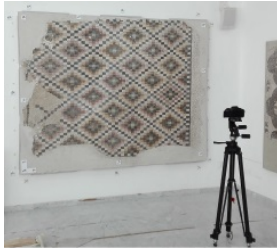

(b)

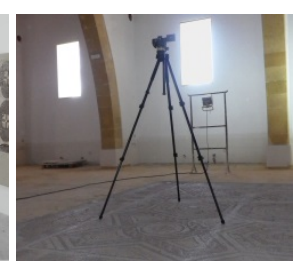

(c)
Figure 5. Data acquisition: (a) Mosaic-1, (b) Mosaic-2 and (c) Mosaic-3.

Table 1. Photogrammetric blocks.

\begin{tabular}{cccc}
\hline Datasets & Images & Nadiral strips & $\begin{array}{c}\text { Convergent } \\
\text { strips }\end{array}$ \\
\hline Mosaic-1 & 401 & 17 & 4 \\
Mosaic-2 & 100 & 8 & 4 \\
Mosaic-3 & 433 & 18 & 4 \\
\hline
\end{tabular}

Some photogrammetric coded targets have been positioned around and along the edge of the mosaics to improve the photo orientation step for the images taken along the edge of the mosaics. The use of coded targets, encoded with a unique identifier, is very common in vision metrology to signalize feature points [25]. Concentric rings coded targets with a 12 bit pattern have been used (Figure 6); these targets are relatively easy to recognize and decode.

It was not possible to measure the position of the targets with accuracy suitable to the purpose of the work but three coded targets were put to define a local reference system (Figure 7); the coordinates of these three targets were arbitrarily

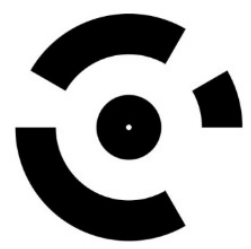

Figure 6. Concentric rings coded targets with a 12 bit pattern.

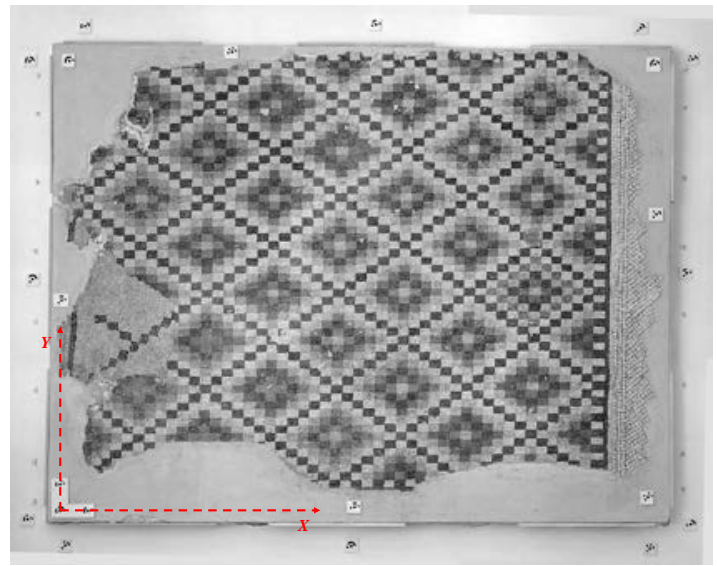

Figure 7. Placement of coded target for Mosaic-2; in the lower left corner the three targets for defining the reference system.

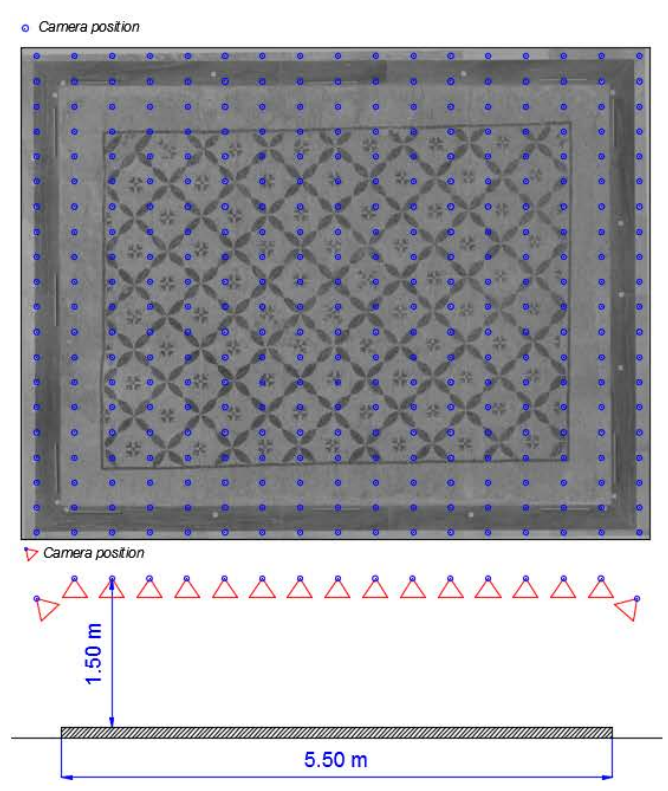

(a)
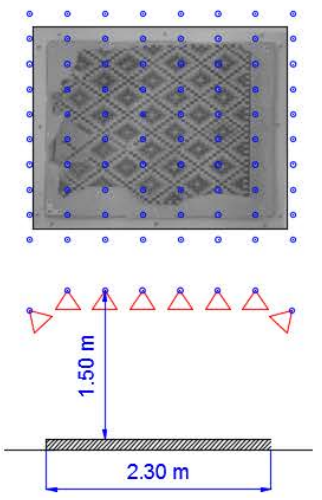

(b)
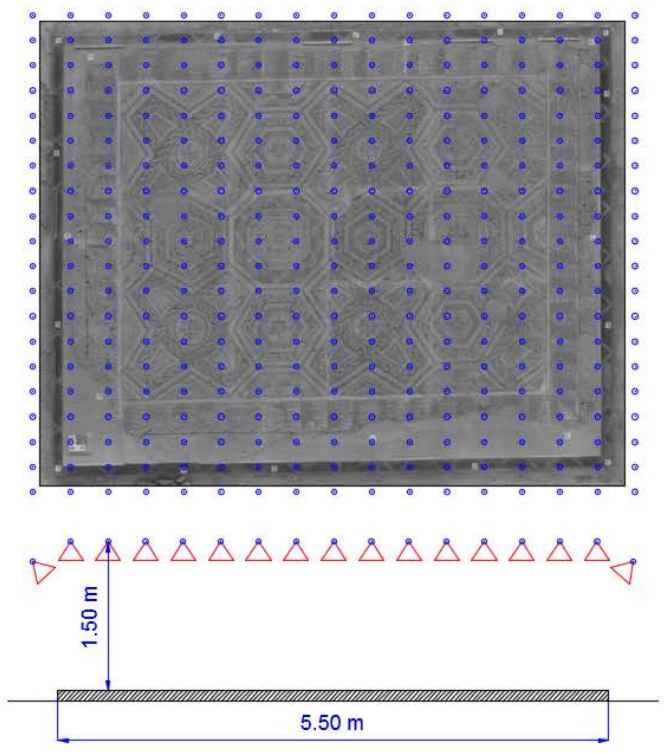

(c)

Figure 4. Planimetric view and cross-section view of camera network geometry for the three datasets: (a) Mosaic-1, (b) Mosaic-2 and (c) Mosaic-3. 
assigned.

Moreover, eleven calibrated bars have been placed along the edge of the mosaics, two near each corner and one along the edge in the center position (only for three edges) (Figure 8). Calibrated bars are of aluminium and are long $50 \mathrm{~cm}$; every bar has four circular holes. The distance between the center of the holes was measured by a computer numerical control machine Mazak Vertical Center Nexus 410a-II with an accuracy of \pm 50 $\mu \mathrm{m}$. For each bar two calibrated distances, one of $48 \mathrm{~cm}$ and another of $46 \mathrm{~cm}$ were measured (Figure 9); one distance has been used to scale the photogrammetric model, the other to check the accuracy of the photogrammetric survey.

To evaluate the theoretical uncertainty of the survey the traditional photogrammetric formulas for so-called "normal case of stereo-photogrammetry" have been used [26]. The theoretical uncertainty $\sigma_{X}$ and $\sigma_{Y}$, respectively in $X$ and $Y$ coordinates, has been calculated from:

$\sigma_{X}=\sigma_{Y}=\frac{D}{c} \sigma_{x^{\prime}}$,

where $D$ is the camera-to-object distance, $c$ is the focal length and $\sigma_{x}$, is the image measurement uncertainty.

The theoretical uncertainty $\sigma_{Z}$ along the $Z$ direction depends also on the ratio $D / B$, where $B$ (baseline) is the distance between the two camera stations, and has been calculated from:

$\sigma_{Z}=\frac{D^{2}}{c \cdot B} \sigma_{p x^{\prime}}$

where $\sigma_{p x}$ is the image measurement uncertainty of the xparallax. These formulas give reasonable approximation of achievable uncertainty and depend primarily on image measurement uncertainty. For a close range photogrammetric/ SfM approach the image measurement uncertainty depends on the accuracy of feature extraction and feature matching, that

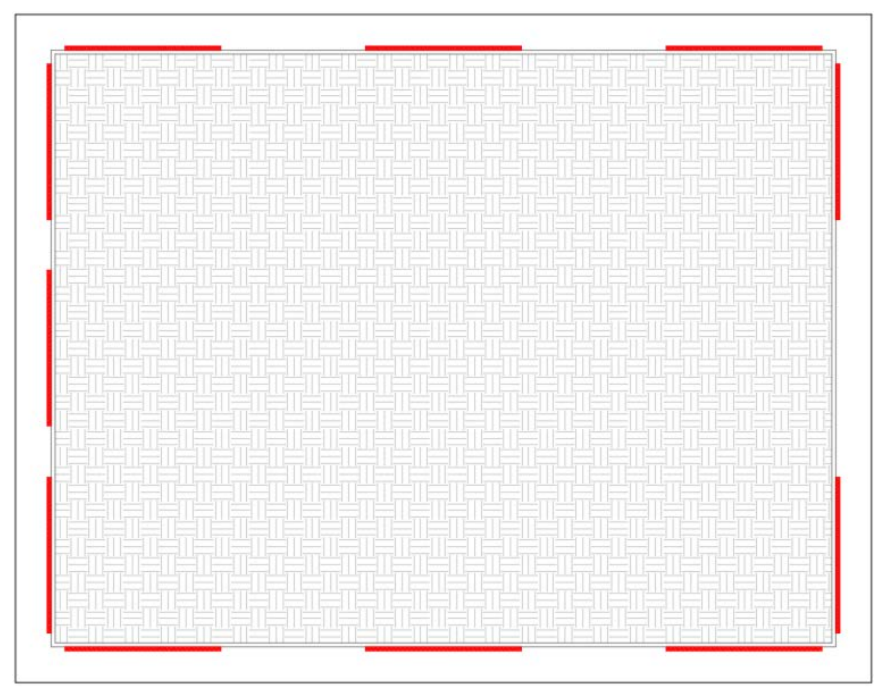

Figure 8. Calibrated bars arrangement along the sides of the mosaic.

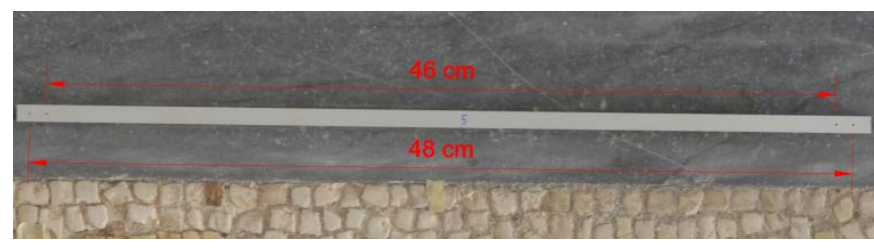

Figure 9. Detail of the calibrated bar. generally can achieve a sub-pixel accuracy [27].

For this work it was supposed $\sigma_{p x^{\prime}}=\sigma_{x^{\prime}}$ and an image measurement uncertainty of 0.5 pixel, corresponding to 1.95 $\mu \mathrm{m}$; the theoretical uncertainty has been estimated at $0.10 \mathrm{~mm}$ for $X$ and $Y$ and $0.42 \mathrm{~mm}$ for the $Z$ direction. The theoretical uncertainty of $2 \mathrm{D}$ point measurement $\sigma_{X Y}$ and $3 \mathrm{D}$ point measurement $\sigma_{X Y Z}$ can be calculated as

$\sigma_{X Y}=\sqrt{\sigma_{X}^{2}+\sigma_{Y}^{2}}$

and

$\sigma_{X Y Z}=\sqrt{\sigma_{X}^{2}+\sigma_{Y}^{2}+\sigma_{Z}^{2}}$.

These values are respectively equal to $0.14 \mathrm{~mm}$ and to $0.44 \mathrm{~mm}$ and, in particular for the $X Y$ accuracy, are compatible with the accuracy of photogrammetric measurements required for the production of an ortho-image at full-scale (scale 1:1). It should be noted that these values are only theoretical values; in fact, the theoretical uncertainty of the so-called "normal case of stereo-photogrammetry" is computed assuming that camera interior orientation parameters and image external orientation parameters are free of error. The actual accuracy depends also on other parameters as the number of images, the metric constraint, etc. and theoretical values must be always validated in the real case.

\section{DATA PROCESSING}

The images processing has been done, through the typical SfM workflow, using the well-known commercial software package PhotoScan Professional Edition by Agisoft LLC [28] (Figure 10). This software package provides a sequence of automatic steps for image orientation and image matching; moreover, PhotoScan allows extracting 3D models and orthoimages with a very high level of detail. During image orientation (called "photo alignment" in PhotoScan) the software can estimate both internal camera parameters and external camera orientation for each image.

PhotoScan is the typical SfM software that has also integrated photogrammetric procedures; in fact, it is possible to recalculate the orientation parameters through a bundle

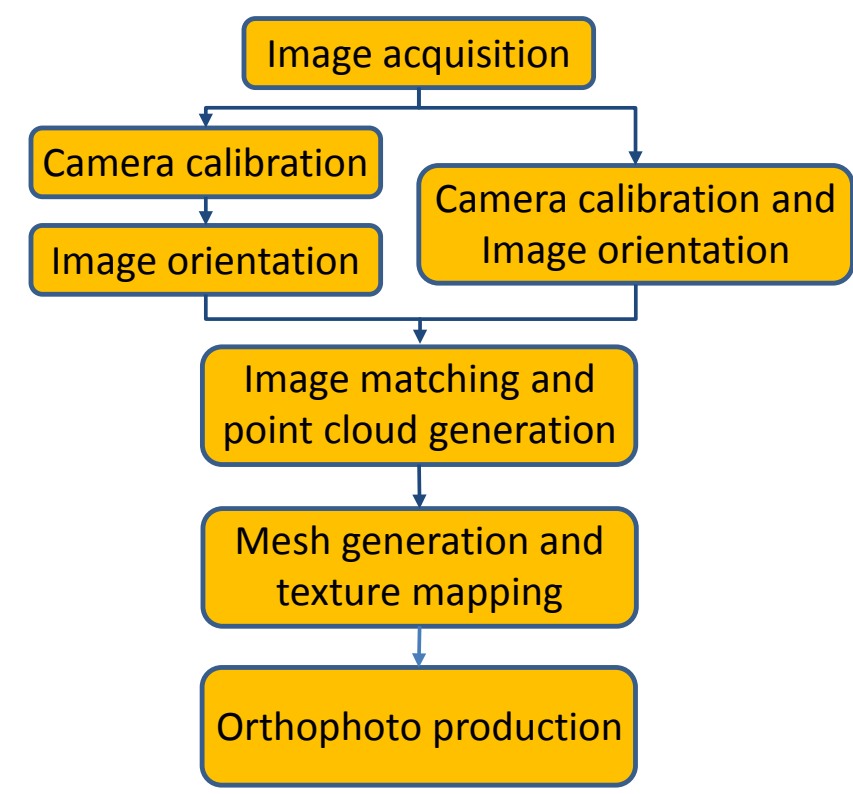

Figure 10. Data processing workflow. 
adjustment with self-calibration (called "optimization" in PhotoScan), to use coded targets for points detection and known distances as metric constraints, to obtain some parameters about the uncertainty of the process and to assign an accuracy value to the metric constraints (ground control points or distances).

For camera calibration the software package PhotoModeler Scanner by EOS Systems Inc. has been used too [29]. PhotoModeler Scanner is a typical and well-known close-range photogrammetry software package.

The data processing was used to test some different camera calibration approaches and to produce very detailed and accurate 3D models and ortho-images.

\subsection{Camera calibration}

The calibration process is one of the most important aspects of the photogrammetric workflow. A correct estimation of camera interior orientation parameters and lens distortion parameters is necessary in order to obtain accurate photogrammetric measurements. This aspect becomes even more important when the accuracy of the photogrammetric survey is less than one millimetre [30]. In close-range photogrammetry the camera is generally calibrated in advance, using a test field, and generally performed with coded targets. The camera interior orientation parameters and the lens distortion parameters were calculated independently from the photogrammetric survey. A self-calibrating bundle adjustment, in which interior orientation parameters and distortion parameters are unknown, was used to solve the camera calibration [31].

According to the SfM approach, the camera calibration process is not considered a particularly important task, and the camera parameters are almost always calculated as part of the orientation process [32]. This approach was developed mainly with the aim of achieving maximum process automation and not the best accuracy, as is the case with photogrammetry. Therefore, camera calibration issues have invariably been not particularly considered. As part of the SfM approach, the use of a huge number of feature points (rather than a small number of targets) can yield enhanced internal uncertainty of the calibration parameters but reduced reliability of the camera parameters. This is mainly due to the poor reliability of the camera network, which may be involved. On the other hand, the high accuracy required for mosaic surveys places great emphasis on camera calibration. For this reason different camera calibration methods were tested to determine the most suitable workflow for this phase. Specifically, two approaches were evaluated: the first used a set of coded targets as part of a typical photogrammetric approach (Pre-Calibration); and the second made use of a standard SfM approach (Self-Calibration) (Figure 11).

The typical photogrammetric approach was performed using either PhotoScan (PS) and PhotoModeler Scanner (PM). Two different test fields were prepared: one with PM-coded targets and another with PS-coded targets (Figure 12). The images were taken in accordance with the classic rules for camera calibration in photogrammetry (multi-station, convergent imaging network, images rotated by $\pm 90^{\circ}$, etc.) [21], thereby obtaining two different calibration datasets for each mosaic. Accordingly, many feature points were measured in the preCalibration process with PS, in addition to the coded targets. Moreover, an additional camera calibration was performed, using the typical SfM approach. The camera parameters were

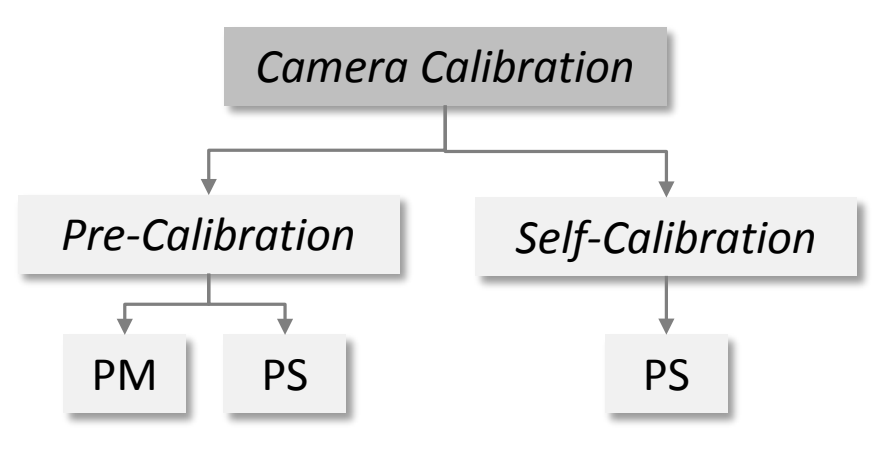

Figure 11. Camera calibration process.

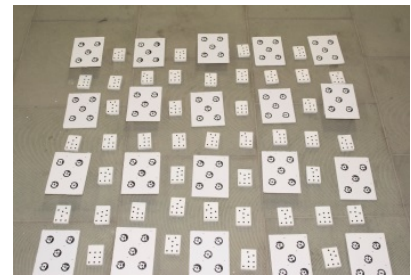

(a)

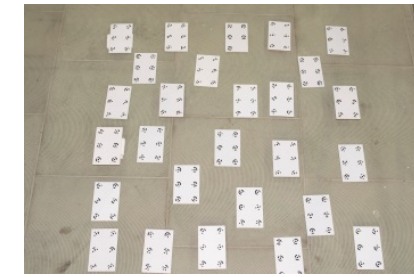

(b)
Figure 12. Camera calibration test field for PM (a) and PS (b).

calculated during the Self-Calibration by the PS optimization process. Three different sets of calibration parameters were, therefore, calculated for each mosaic (PM Pre-Calibration, PS Pre-Calibration and PS Self-Calibration).

The standard photogrammetric camera model for camera calibration, which consists of three elements of interior orientation (principal point coordinates and principal distance) and three types of lens distortion (radial, tangential and affine), was taken into consideration in the analysis.

After this process, all parameters were analysed according to the camera model for PS and the parameters which had been calculated with PM were converted into the PS standard, using the Agisoft Lens software package. This software is necessary because various software packages use slightly different camera models and parameter sets for their calibrations; the parameters are not, however, directly comparable. The camera model used in PS assume a central projection camera where non-linear distortions are modelled using the self-calibration model proposed by Brown [33]. A camera model specifies the transformation from point coordinates in the local camera coordinate system to the pixel coordinates in the image frame. The model used to project points in the local camera coordinate system is

$x=\frac{X}{Z}$

$y=\frac{Y}{Z}$

$r=\sqrt{x^{2}+y^{2}}$

$x^{\prime}=x\left(1+K_{1} r^{2}+K_{2} r^{4}+K_{3} r^{6}+K_{4} r^{8}\right)+\left(P_{1}\left(r^{2}+\right.\right.$

$\left.\left.2 x^{2}\right)+2 P_{2} x y\right)\left(1+P_{3} r^{2}+P_{4} r^{4}\right)$

$y^{\prime}=y\left(1+K_{1} r^{2}+K_{2} r^{4}+K_{3} r^{6}+K_{4} r^{8}\right)+\left(P_{1}\left(r^{2}+\right.\right.$

$\left.\left.2 y^{2}\right)+2 P_{2} x y\right)\left(1+P_{3} r^{2}+P_{4} r^{4}\right)$

$u=\frac{w}{2}+x_{p}+x^{\prime} c+x^{\prime} B_{1}+y^{\prime} B_{2}$

$v=\frac{h}{2}+y_{p}+y^{\prime} c$, 
where $(\mathrm{X}, \mathrm{Y}, \mathrm{Z})$ are the point coordinates in the local camera coordinate system, $(u, v)$ are the projected point coordinates in the image coordinate system (in pixels), $c$ is the principal distance, $\left(x_{p}, y_{p}\right)$ are the principal point coordinates, $K_{n}$ are the radial distortion parameters, $P_{n}$ are the tangential distortion parameters, $B_{n}$ are the affinity and shear distortion parameters, and $(w, b)$ are the image width and height in pixels.

Two procedures can be followed in determining the most reliable values of camera calibration: the first concerns the recovery accuracy of the individual parameters of the calibration model, while the second relates to the accuracy impact on any subsequent photogrammetric measurements, i.e. the effect of calibration errors on object point determination [34]. The first approach could be applied to close-range photogrammetric software packages (like PM), which reports the standard error of the unknown parameters computed during the camera calibration. These errors can provide us with information regarding the inner reliability of the process but it does not give any information about the accuracy of the object point coordinates.. Moreover, the software package from a computer vision, like PS, lacks information regarding this step. For these reasons, it is not possible to evaluate the camera calibration according to the first procedure. The second procedure requires various independent checking measures to estimate the accuracy in object space and to evaluate camera calibration reliability. This second procedure will be described in detail in Subsection 4.2. The values obtained in the calibration with the test field (Pre-Calibration) are always very similar to each other. On the other hand, the values obtained by self-calibration are different from each other, particularly regarding the principal distance and principal point coordinates.

Moreover there exist differences in the lens distortion coefficients (on occasions of one or two orders of magnitude), specifically, the radial distortion parameters $\mathrm{K} 3$ and $\mathrm{K} 4$ and the affine distortion coefficients B1 and B2.

\subsection{Image orientation}

Images were oriented with the aim of assessing the most suitable camera calibration procedure with which to obtain the maximum accuracy of the final products. Three different projects were created for each dataset: one with the parameters calculated by PM (PM Pre-Calibration project), another with the parameters calculated by PS (PS Pre-Calibration project) and the third with the parameters calculated by PS during image orientation (PS Self-Calibration project). Images were oriented using only PS and this procedure was divided into several steps: i. image orientation with an SfM approach, which was performed considering the first 1/16 of the original image resolution (low photo alignment), without any image pair preselection for feature points detection. Thereafter, considering $1 / 2$ of the original image resolution (high photo alignment) and the generic preselection mode, which only used the overlapping pairs of photos to detect feature points (no camera calibration parameters were estimated at this stage)

ii. an automatic measurement of the coded targets and manual measurement of the calibrated bars

iii. importing a different set of camera calibration parameters, which had been held constant throughout the next step (no parameters were imported in the PS Self-Calibration project)

iv. a re-computation of exterior orientation parameters by means of a bundle adjustment, taking the calibrated bars into consideration as constraints. In this step the camera calibration parameters were also calculated in the PS Self-Calibration project

v. an analysis of the results.

All the projects were calculated according to an arbitrary reference system by applying a free-network solution bundle block adjustment and by using only the $48 \mathrm{~cm}$ calibrated distances of the scale bars with which to scale the photogrammetric model. However, the $46 \mathrm{~cm}$ calibrated distances of the scale bars were used as independent check, control distances with which to evaluate the accuracy of the projects. This procedure was chosen in agreement with German standards (VDI/VDE 2634 Part 1) [15] for evaluating the object space accuracy of 3D point measurement systems, which are based on a length measurement error (LME). This value is the difference between the distance calculated by the photogrammetric process and the calibrated distance of the scale bar; the root mean square error (RMSE) of all the differences provides us with an assessment of the accuracy of the photogrammetric measurement.

Table 2 reports the RMSE of the scale bars for $48 \mathrm{~cm}$ calibrated distances, which were used to scale the photogrammetric model, and the $46 \mathrm{~cm}$ calibrated distances, which were used as an independent check. The mean of the residuals was also checked to verify the presence of systematic effects. The mean of the residuals for all projects was very close to zero for the distances used to scale the photogrammetric model and for distances used as an independent check. The RMSE of the calibrated check distances for almost all projects was lower than $0.2 \mathrm{~mm}$. The diverse tests indicated that the

Table 2. RMSE for scale bars.

\begin{tabular}{|c|c|c|c|c|}
\hline & & $\begin{array}{c}\text { PM } \\
\text { Pre-Calibration } \\
{[\mathrm{mm}]} \\
\end{array}$ & $\begin{array}{c}\text { PS } \\
\text { Pre-Calibration } \\
{[\mathrm{mm}]} \\
\end{array}$ & $\begin{array}{c}\text { PS } \\
\text { Self-Calibration } \\
{[\mathrm{mm}]}\end{array}$ \\
\hline \multirow{2}{*}{ Mosaic-1 } & $\begin{array}{c}\text { RMSE } \\
\text { Scale distances }\end{array}$ & 0.453 & 0.050 & 0.030 \\
\hline & $\begin{array}{c}\text { RMSE } \\
\text { Check distances }\end{array}$ & 0.440 & 0.054 & 0.033 \\
\hline \multirow[t]{2}{*}{ Mosaic-2 } & $\begin{array}{c}R M S E \\
\text { Scale distances }\end{array}$ & 0.137 & 0.083 & 0.230 \\
\hline & $\begin{array}{c}\text { RMSE } \\
\text { Check distances }\end{array}$ & 0.118 & 0.068 & 0.180 \\
\hline \multirow[t]{2}{*}{ Mosaic-3 } & $\begin{array}{c}\text { RMSE } \\
\text { Scale distances }\end{array}$ & 0.158 & 0.181 & 0.183 \\
\hline & $\begin{array}{c}R M S E \\
\text { Check distances }\end{array}$ & 0.162 & 0.180 & 0.191 \\
\hline
\end{tabular}


projects with camera calibration parameters obtained as part of an independent process could provide greater accuracy. There was a notable improvement in Mosaic 2 (of approximately one order of magnitude for the PS Pre-Calibration project) while the differences with Mosaic 3 were less evident. Only Mosaic 1 revealed different results: very high residuals for the PM PreCalibration project (the calibrated check distances reached about $0.4 \mathrm{~mm}$ ) but very low residuals (in the order of few hundredths of a millimetre) for the other two projects.

The accuracy evaluation has, however, some critical issues. The arrangement of the calibrated bars is not the most favourable because the bars are only along the edges of the mosaics. Theoretically, they should be placed also inside the mosaic; this condition was not possible because in this way they would cover the tesserae of the mosaic. The accuracy verification should be performed along three dimensions, as required in the VDI/VDE Guideline; this was challenging for a survey of mosaics and the scale bar distribution was indeed coplanar with the mosaics. Finally, the dimension of the mosaic would have required some more long reference distances, similar to the dimension of the mosaics (i.e. $4 \mathrm{~m}$ or $5 \mathrm{~m}$ ). In our study, the short calibrated distance was due to the limit of the computer numerical control machine used for the distance measurement. This issue is generally very common in metrology applications with close-range photogrammetry; in fact, it is very difficult to find scale bars suitable for large measuring objects (dimension $>3 \mathrm{~m}$ ) [26]. For large measuring objects, accuracy evaluation could be checked using very accurate checkpoints measured with total station that allowed recording data with high angular and distance accuracy, and with very redundant topographic schemes. This approach is not always possible because of the logistical conditions of the site, such as in our situation where the photogrammetric surveys were conducted in museums.

As can be noted from Table 2, the accuracy obtained in almost all tests was better than the theoretical 3D uncertainty $\sigma_{X Y Z}$, which had been calculated in (4); however, as previously underlined, the scale bars arrangement is not the most suitable for assessing 3D accuracy. The results are comparable with the theoretical $2 \mathrm{D}$ uncertainty $\sigma_{X Y}$, which was calculated in (3). The accuracy results could however be considered as satisfactory in obtaining a full-scale (scale 1:1) ortho-image.

\section{3D MODELS AND ORTHO-IMAGES OF ANCIENT MOSAICS}

The 3D models and the ortho-images of the ancient mosaics were obtained using the projects which obtained the greatest accuracy (that is, the PS Pre-Calibration projects for Mosaic 1 and Mosaic 2, PM Pre-Calibration for Mosaic 3). The 3D models were achieved according to the following steps: calculating of a dense point cloud by dense image matching, building of a 3D mesh and texturing of the 3D mesh.

The point clouds were calculated by taking into account: the theoretical GSD, the residuals of the orientation phase and the level of detail suitable for the 3D model. Thus, a point cloud with a $1 / 4$ of an image resolution was calculated as corresponding to about 0.8 millimeters (Figure 13). Three point clouds with 74 million points (Mosaic 1), 16 million points (Mosaic 2) and 63 million points (Mosaic 3) were obtained, and three meshes with 15 million polygons (Mosaic 1), 3 million polygons (Mosaic 2) and 12 million polygons (Mosaic 3) were calculated respectively from the point clouds. The RGB value was also associated with the $3 \mathrm{D}$ position for each point during the $3 \mathrm{D}$ reconstruction process. This value in the mesh generation process permitted the assignment of a mean value of RGB to each polygon. The more defined the mesh, in terms of numbers of polygons and geometry, the better the appearance of the 3D model. Generally, a mesh with a coloured vertex will be sufficient in providing an approximate idea of the condition of the mosaic and the type of material from which it was made. In order to increase this level of detail, it is necessary to provide texture to the 3D model with the images of the photogrammetric survey. Five textures of 4,096 pixels $\times 4,096$ pixels each were generated for each 3D model with the texture number ensuring the resolution of a sufficiently detailed 3D model to analyze even the smallest details (Figure 14).

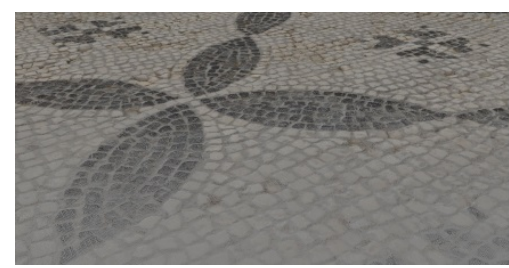

(a)

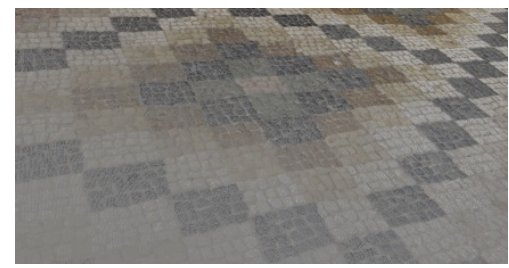

(b)

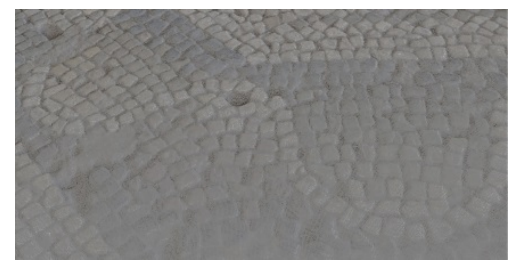

(c)

Figure 13. Detail of Mosaic-1 (a), Mosaic-2 (b) and Mosaic-3 (c) point clouds.

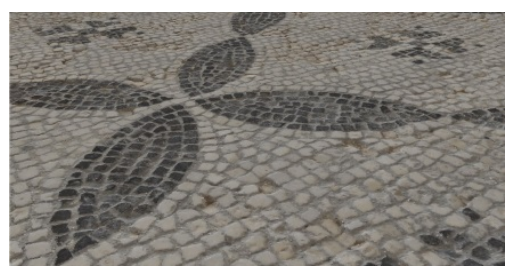

(a)

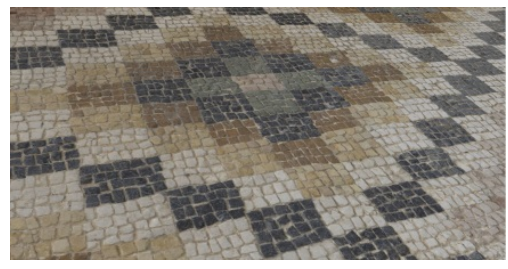

(b)

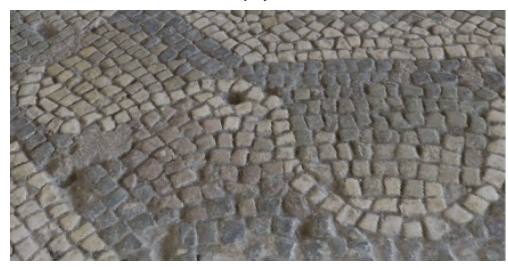

(c)

Figure 14. Detail of Mosaic-1 (a), Mosaic-2 (b) and Mosaic-3 (c) 3D models. 
Finally, the ortho-images of the three mosaics were calculated with a geometric resolution of $0.5 \mathrm{~mm}$ (Figure 15). Good chromatic continuity and enhanced definition of the details of the mosaics were obtained in all the cases under investigation. Despite being 2D data, ortho-images still constitute the main type of documentation of single tessera: they allow us to examine numerous irregularities, the size and position of an individual tessera and the presence of erosion and patina deterioration with non-invasive and more efficient procedures.

\section{CONCLUSIONS}

The research outline in this paper has evaluated the surveying of mosaics using a photogrammetric/computer vision approach in the context of vision metrology. Different camera calibration processes have been tested in order to identify the most appropriate workflow.

The research was performed on three ancient Roman mosaics as test data. Aspects relating to the camera calibration process were evaluated by virtue of different sets of internal orientation parameters. Moreover, the camera calibration parameters were calculated in accordance with two photogrammetric test fields (performed with coded targets) and a SfM approach.

The tests revealed slight changes between the camera parameters, particularly for the principal distance and for principal point coordinates. An accuracy evaluation was conducted by the residuals obtained from various scale bars, which were used as checks. These results also demonstrate that the projects with camera calibration parameters, which had been obtained via an independent process, could offer enhanced accuracy. Differences among the different datasets were also observed. However, an RMSE of the calibrated check distances for almost all projects was observed to be lower than $0.2 \mathrm{~mm}$.

The survey facilitated the obtaining of a $3 \mathrm{D}$ reconstruction and an ortho-image for each of the three mosaics, which was compatible with one at full scale (scale 1:1). This type of documentation could be repeated over the time to monitor and analyse mosaics even after restoration processes. The high accuracy obtained with a photogrammetric/SfM approach demonstrates that these techniques could also be applied to a context of vision metrology relating to archaeological 3D documentation. An evaluation of the accuracy of this approach also confirms that camera calibration is still an open issue and additional tests are required to improve any evaluation of the accuracy of the photogrammetric/SfM approach.

\section{ACKNOWLEDGEMENT}

Thanks to Directors of Antonino Salinas Regional Archaeological Museum in Palermo and Baglio Anselmi Regional Archaeological Museum in Marsala for allowing the execution of the surveys.

Thanks to Maria Grazia Spera for the help in data processing and in the survey of the Mosaic 2, to Lorella Pellegrino, Alessandra Garraffa, Barbara Di Natale for the collaboration in the study and in the surveys of Mosaic 1 and Mosaic 3, and also to Enza Mancuso for the help in the survey of Mosaic 2.

\section{REFERENCES}

[1] G. Guidi, F. Remondino, "3D Modelling from real data". In Modelling and Simulation in Engineering, Edited by Catalin Alexandru, InTech, 2012, pp. 69-102.

[2] F. Remondino, S. El-Hakim, "Image-based 3D modelling: a review", The Photogrammetric Record, vol.21(115), 2006, pp. 269-291.

[3] M. Doneus, G. Verhoeven, M. Fera, C. Briese, M. Kucera, W. Neubauer, "From deposit to point cloud: a study of low-cost computer vision approaches for the straightforward documentation of archaeological excavations". In K. Pavelka (editor), Geoinformatics (Faculty of Civil Engineering, Czech Technical University in Prague), vol. 6, 2011, pp. 81-88.

[4] T.P. Kersten, M. Lindstaedt, "Image-Based Low-Cost Systems for Automatic 3D Recording and Modelling of Archaeological Finds and Objects". In: Ioannides M., Fritsch D., Leissner J., Davies R., Remondino F., Caffo R. (eds) Progress in Cultural Heritage Preservation. EuroMed 2012. Lecture Notes in Computer Science, Springer, vol 7616, 2012, pp. 1-10.

[5] C. Santagati, L. Inzerillo, F. Di Paola, "Image-based modelling techniques for architectural heritage 3D digitalization: limits and potentialities", Int. Arch. Photogramm. Remote Sens. Spatial Inf. Sci., XL-5/W2, pp. 555-560.

[6] J. De Reu, G. Plets, G. Verhoeven, P. De Smedt, M. Bats, B. Cherretté, W. De Maeyer, J. Deconynck, D. Herremans, P. Laloo, M. Van Meirvenne, W. De Clercq, "Towards a threedimensional cost-effective registration of the archaeological heritage", Journal of Archaeological Science, vol. 40, issue 2, 2013, pp. 1108-1121.

[7] S. Green, A. Bevan, M. Shapland, "A comparative assessment of structure from motion methods for archaeological research", Journal of Archaeological Science, vol. 46, 2014, pp. 173-181.

[8] A. Koutsoudis, B. Vidmar, G. Ioannakis, F. Arnaoutoglou, G. Pavlidis, C. Chamzas, "Multi-image 3D reconstruction data

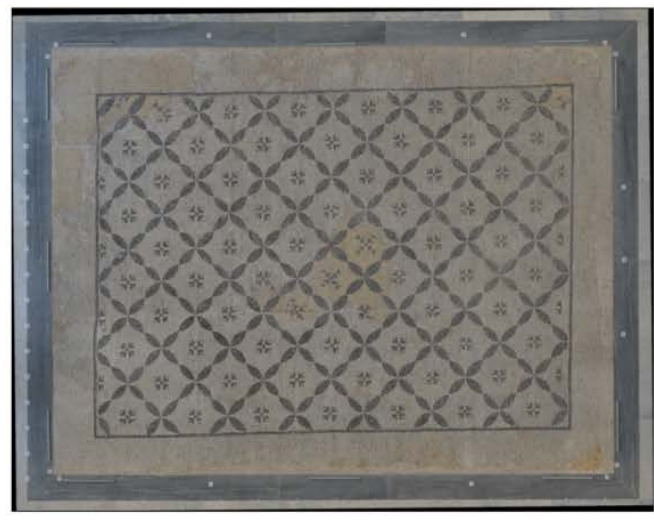

(a)

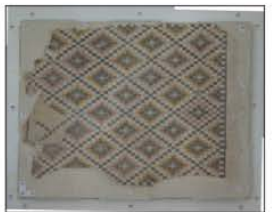

(b)

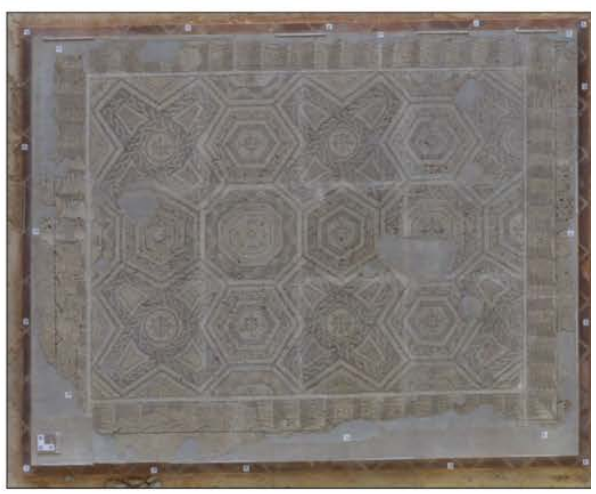

(c)

Figure 15. Mosaic 1 (a), Mosaic 2 (b) and Mosaic 3 (c) ortho-images. 
evaluation", Journal of Cultural Heritage, vol.15(1), 2014, pp. 73 79 .

[9] C.S. Fraser, "Innovations in automation for vision metrology systems”, Photogrammetric Record, vol. 15(90), 1997, pp. $901-$ 911.

[10] S. Robson, M. Shortis, "Engineering and manufacturing", in Application of 3D measurement from images, ed. by J. Fryer, $\mathrm{H}$. Mitchell, J. Chandler, Whittles Publishing, 2007, pp. 65-101.

[11] N. Mostofi, F. Samadzadegan, Sh. Roohy, M. Nozari, "Using vision metrology system for quality control in automotive industries", Int. Arch. Photogramm. Remote Sens. Spatial Inf. Sci., XXXIX-B5, 2012, pp. 33-37.

[12] F. Di Paola, T. Ingrassia, M. Lo Brutto, A. Mancuso, "A reverse engineering approach to measure the deformations of a sailing yacht", Advances on Mechanics, Design Engineering and Manufacturing, Part of the series Lecture Notes in Mechanical Engineering, Springer International Publishing, 2016, pp. 555563.

[13] M. Lo Brutto, D. Termini, "Bed topography reconstruction in a large amplitude maendering flume: application of close range photogrammetry". Advanced Materials Research, Trans Tech Publications, Switzerland, vol. 875-877, 2014, pp. 440-444.

[14] G.D. Gessesse, H. Fuchs, R. Mansberger, A. Klik, D.H. RiekeZapp, "Assessment of Erosion, Deposition and Rill Development On Irregular Soil Surfaces Using Close Range Digital Photogrammetry". The Photogrammetric Record, vol. 25 2010, pp. 299-318.

[15] VDI/VDE Guideline 2634 Part 1, "Optical 3D measuring systems - Imaging systems with point-by-point probing”, Beuth Verlag, Berlin, Germany, 2002.

[16] I. Toschi, E. Nocerino, M. Hess, F. Menna, B. Sargeant, L. MacDonald, F. Remondino, S. Robson, "Improving automated 3D reconstruction methods via vision metrology", Proc. of Videometrics, Range Imaging and Applications XIII, SPIE Optical Metrology, Vol. 9528, 2015.

[17] R. Szeliski, "Computer Vision: Algorithms and Applications", Springer: Berlin/Heidelberg, Germany, 2010.

[18] I. Toschi, A. Capra, L. De Luca, J.A. Beraldin, L. Cournoyer, "On the evaluation of photogrammetric methods for dense 3D surface reconstruction in a metrological context", ISPRS Ann. Photogramm. Remote Sens. Spatial Inf. Sci., II-5, 2014, pp. $371-$ 378.

[19] M.J. Westoby, J. Brasington, N.F. Glasser, M.J. Hambrey, J.M. Reynolds, "Structure-from-Motion photogrammetry: A low-cost, effective tool for geoscience applications", Geomorphology, vol. 179, 2012, pp. 300-314.

[20] L. Barazzetti, F. Remondino, M. Scaioni, M. Lo Brutto, A Rizzi, R. Brumana, "Geometric and radiometric analisys of paintings".
Int. Arch. Photogramm. Remote Sens. Spatial Inf. Sci., XXXVIII, Part. 5, 2010, pp. 62-67.

[21] D. Abate, F. Menna, F. Remondino, M.G. Gattari, “3D painting documentation: evaluation of conservation conditions with $3 \mathrm{~d}$ imaging and ranging techniques", Int. Arch. Photogramm. Remote Sens. Spatial Inf. Sci., XL-5, 2014, pp. 1-8.

[22] L. Fregonese, C.C. Monti, G. Monti, L. Taffurelli, “The St Mark's Basilica Pavement: The Digital Orthophoto 3D Realisation to the Real Scale 1:1 for the Modelling and the Conservative Restoration", Innovations in 3D Geo Information Systems, First International Workshop on 3D Geoinformation, 7-8 August 2006, Kuala Lumpur, Malaysia, 2006.

[23] O. Ajioka, Y. Hori, "Application of SfM and laser scanning technology to the description of mosaics piece by piece", Int. Arch. Photogramm. Remote Sens. Spatial Inf. Sci., XL-5, 2014, pp. 23-28.

[24] E. Nocerino, F. Menna, F. Remondino, "Accuracy of typical photogrammetric networks in cultural heritage 3D modeling projects", Int. Arch. Photogramm. Remote Sens. Spatial Inf. Sci., XL-5, 2016, pp. 465-472.

[25] U. Wijenayake, S. I. Choi, S. Y. Park, "Automatic detection and decoding of photogrammetric coded targets", International Conference on Electronics, Information and Communications (ICEIC), Kota Kinabalu, 2014, pp. 1-2.

[26] T. Luhmann, S. Robson, S. Kyle, I. Harley, "Close Range Photogrammetry: Principles, Techniques and Applications", Whittles Publishing, 2011.

[27] K. Mikolajczyk, C. Schmid, "A performance evaluation of local descriptors". IEEE Trans. Pattern Anal. Mach. Intell., 27, 2005, pp. 1615-1630.

[28] http://www.agisoft.com/

[29] http://www.photomodeler.com/index.html

[30] T. Luhmann, "Close-Range Photogrammetry for Industrial Applications". ISPRS Journal of Photogrammetry and Remote Sensing, 65(6), 2010, pp. 558-569.

[31] F. Remondino, C. Fraser, "Digital camera calibration methods: considerations and comparisons". Int. Arch. Photogramm. Remote Sens. Spatial Inf. Sci., XXXVI, part 5, 2006, pp. 266-272.

[32] T. Luhmann, C. Fraser, H.G. Maas, "Sensor modelling and camera calibration for close-range photogrammetry", ISPRS Journal of Photogrammetry and Remote Sensing, 115, 2016, pp. 37-46.

[33] D. C. Brown, "Close-range camera calibration". Photogrammetric Engineering, vol. 37(8), 1971, pp. 855-866.

[34] S.K. Nouwakpo, M. R. James, M. A. Weltz, C.-H. Huang, I. Chagas, L. Lima, "Evaluation of structure from motion for soil microtopography measurement". Photogrammetric Record, vol. 29, 2014, pp. 297-316. 